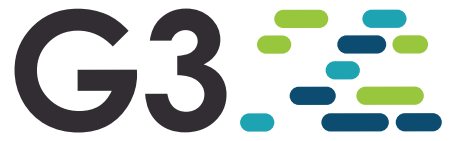

Genes | Genomes | Genetics

\title{
The Genetic Diversity of Two Subpopulations of Shenxian Pigs as Revealed by Genome Resequencing
}

\author{
Liu Diao*,1, Cao Hongzhan*,1,2, Lu Chunlian*,3, Li Shang*,4, Jia Mengyu*,5, Li Sai ${ }^{\dagger}$ and Ren Liqin $\neq$ \\ ${ }^{*}$ College of Animal Science and Technology, Hebei Agricultural University, Baoding 071000, Hebei, ${ }^{\dagger}$ Hebei Zhengnong Animal Husbandry Co., Ltd., Xinji \\ 052360, Hebei, ‡Fishery Technology Extension Station, Wanquan District, Zhangjiakou City, Zhangjiakou, Hebei 076250
}

ABSTRACT Shenxian pigs can be divided into two main strains from their shape and appearance: HuangKEYWORDS

\section{INTRODUCTION}

Shenxian pig is the only outstanding local pig breed listed in the "Chinese Pig Breeds" in Hebei Province. Compared with other imported pig breeds, Shenxian pigs have an earlier sexual maturity, short estrus interval, and a large number of litters. They have good research significance. There are two main strains of Shenxian pigs: Cucumber beak and Wuhuatou. "Cucumber Mouth": The mouth is long and pointed at the end, hence the name. The nose is straight and flat, with few hairs, shallow wrinkles on the forehead, slender neck, narrow forequarters, long and concave back and waist, slightly drooping abdomen, inclined buttocks, weak hind limbs, and thick tail. "Five-flowered head": The head is rough, the hair is thick, the forehead is wide and wrinkled, and it gets its name. Based on the whole-genome resequencing technology, the genetic structure of the two sub-populations of Shenxian pigs was discussed, and the evolutionary mechanism between the two populations was revealed at the molecular level, and all genetic

Manuscript compiled: Saturday $23^{\text {rd }}$ January, 2021

${ }^{1}$ These authors contributed equally to this work.

${ }^{2}$ These authors contributed equally to this work.

${ }^{3}$ Corresponding author: College of Animal Science and Technology, Hebei Agricultural

University, Baoding 071000.E-mail:chz516@126.com information in the whole genome was accurately obtained, and the two populations were discovered to the greatest extent. Genetic variation(Bi et al.2020).

\section{MATERIALS AND METHODS}

\section{Materials}

The pig farm in Shenxian County was selected from Hebei Zhengnong Animal Husbandry Co., Ltd. and selected healthy, weight, and age-similar cucumber beaks and 5 pigs with fiveflower head each. Cucumber beaks: B1-1, B1-2, B1-3, B1 -4, B1-5, five flower head: B2-1, B2-2, B2-3, B2-4, B2-5. Use ear tag tongs to collect ear tissue, shear pig ear tissue and disinfect with 75 percent alcohol, take about 100mg sample, store the sample in an EP tube containing 95 percent alcohol, transport it back to the laboratory with an ice pack, and store it in a refrigerator at $-20^{\circ} \mathrm{C}$ It is reserved for use in sampling, transportation and storage to ensure that the samples are not contaminated for subsequent extraction of genomic DNA.

\section{Whole genome resequencing}

Whole genome resequencing is process used to sequence the genomes of different individuals of species with known genome sequences, and then analyze the differences of individuals or groups 
on that basis(Ley et al.2008). It has been found that based on whole genome resequencing technology, researchers have been able to quickly carry out resource surveys and screening processes in order to determine large numbers of genetic variations, and realize genetic evolution analyses and predictions of important candidate genes. In the current study, the two subpopulations of Shenxian pigs were re-sequenced for the purpose of obtaining the genomic information. A large number of high accuracy SNPs, InDel, and other variation information were obtained by comparing the results with the reference genome. Subsequently, the variation information was successfully detected, annotated, and counted.

\section{Detection methods based on next generation sequencing tech- nology}

At present, the second-generation sequencing technology is widely used(Levy et al.2016;Slatko et al.2018). The development of the next generation sequencing (NGS) technology has resulted in revolutionary changes in the detections of genetic variations. Researchers can now comprehensively and accurately detect various types and the sizes of genetic variations from the genomic level(Mardis.2008) For the detection of genetic variations based on the next generation sequencing technology, the first step is to compare the reading segments of thousands of sequences to the corresponding positions on the reference genome. Then, the possible variation information can be inferred using simple mathematical models(Andersson.2009;Mckenna et al.2010;Montgomery et al.2013). These methods take the number of reading segments supporting the variations and reference sequences; quality of the reading segment comparison; environmental conditions of the genome sequences; and the possible noise (such as sequencing errors) as prior information. Then, the variation types and genotypes at certain positions can be determined according to naive Bayesian theory, and the genotype with the highest posterior probability can be successfully obtained(Montgomery et al.2013;Durbin et al.2008). Such methods have been found to be effective for SNP and small InDel detections(Depristo et al.2011;Albers et al.2011).

\section{Data processing}

This test sample uses the whole genome resequencing data (sequencing depth 10X) of 10 Shenxian pigs ( 5 cucumber-mouthed pigs and 5 Wuhuatou pigs). The output of the genome data comparison is the sam file. Since the sorting method of the sam file cannot be used for subsequent analysis, the Samtools software(Li et al.2009)is used to convert the sam file into a file in chromosome sorting format, that is, the bam format. Chromosome rearrangement is to arrange the entries of the same chromosome in the file in ascending order, and merge the two files of the same sample.

SNP detection and annotation: Use GATK and VarScan software(Koboldt et al.2009)to perform SNP detection at the same time, so as to ensure that the obtained SNP site information will not be affected by the deviation of base misalignment caused by InDel mutation. GATK detection SNP code: java -jar GenomeAnalysisTK.jar glm SNP -R ref.fa -T UnifiedGenotyper -I test.sorted.repeatmark.bam -o test.raw.vcf. In order to ensure the accuracy of SNP information, strict testing conditions are set when detecting SNP site information: $<1>$ The minimum number of end reads greater or equal to $4 ;<2>$ The minimum quality value Q20 greater or equal to $90 ;<3>$ Minimum coverage greater or equal to $6 ;<4>\mathrm{P}$ value Less than or equal to 0.01 . InDel detection and annotation: The same use of GATK and VarScan software for InDel detection can eliminate errors caused by SNP variation. GATK detection InDel code: java -jar GenomeAnalysisTK.jar glm
InDel -R ref.fa - T UnifiedGenotyper -I test.sorted.repeatmark.bam -o test.raw.vcf. The detection conditions are consistent with SNP. The detected SNP and InDel mutation sites also need quality control, remove untrusted data sites, correct the quality value, and output the file in VCF format after correction. Use R language(Gao et al.2014;Sudhaka.2018)and ANNOVAR software (Kai et al.2014)to perform mutation information Sort and comment.

\section{RESULTS AND DISCUSSION}

\section{Results}

BWA software (Version: 0.7.15-r1140) was used in this research investigation to compare the clean sequences of the two subpopulations of Shenxian pigs after quality control with the latest version of reference genome of pigs was implemented. The results are detailed in Table 2-1. It was found that on average, more than 86 percent of the sequencing data could be compared to the reference genome.

There are a large number of SNPs, and there may be one SNP variant site every 500-1000bp(Group et al.2001).Count the gene region and type of SNP to get Table 1.A total of 14509223 SNPs were detected in Huangguazui pigs (B1), of which there were 88176.4 $(0.61 \%)$ and $101948.2(0.70 \%)$ in the upstream and downstream $1 \mathrm{~kb}$ region of the gene, and $7865381(54.21 \%)$ in the intergenic region. There are an average of $99217.4(0.68 \%)$ and $6151792(42.40 \%)$ of exons and introns. A total of 13660201 SNPs were detected in Wuhuatou pig (B2), of which 84855.2 (0.62\%) and $97797.2(0.72 \%)$ were found in the upstream $1 \mathrm{~kb}$ region and the downstream $1 \mathrm{~kb}$ region of the gene, and the intergenic region contained 7407500 $(54.23 \%)$. There are $96530.8(0.71 \%)$ and $5782023(42.33 \%)$ of exons and introns respectively.

In the current study, the SNP was identified using GATK software, and the SNP data were subsequently analyzed. The original data contained a total of ten samples and 31,658,350 SNP sites. Effective data was obtained after a secondary quality control of the original data, with ten samples and 23,088,823 SNP sites remaining. The total samples contained the individuals of the two strains of Shenxian pigs.A total of 580 high-quality polymorphic SNPs were obtained by screening $23,088,823$ SNP sites. The marker covered eleven chromosomes, and its PIC index ranged from 0.1 to 0.529 , with an average value of 0.305 . There were 119 highly polymorphic SNPs and 191 moderately polymorphic SNPs identified.In this study, the quantity and density distributions of the SNPs on chromosomes were further analyzed. The results are shown in Figure 1. The distributions of the quantities and densities of the SNPs on the chromosomes were observed to not be uniform. For example, Chromosome 15 was short in length but had the largest number of SNPs, which was far more than the other examined chromosomes. These findings indicated that the densities of the SNPs on that particular chromosome were the highest. Therefore, it was considered that there was a relatively high density of variation on Chromosome 15.

In order to infer the genetic relationship between the Huangguazui and Wuhuatou pig subpopulations, a genetic distance matrix of the IBS was constructed using Plink (V1.9) software(Purcell et al.2007). Then, based on the results, a phylogenetic tree was constructed using a neighbor joining method (NJ), as detailed in Figure 2. The analysis results showed that the Huangguazui and Wuhuatou subpopulations had originated from the same ancestor and belonged to the same variety. However, they could clearly be divided into two distinct groups, which indicated that there were indeed differences in the genetic backgrounds of the Huangguazui and Wuhuatou pigs. It was confirmed that there was a certain 
bioRxiv preprint doi: https://doi.org/10.1101/2021.01.25.428118; this version posted January 26, 2021. The copyright holder for this preprint (which was not certified by peer review) is the author/funder. This article is a US Government work. It is not subject to copyright under 17 USC 105 and is also made available for use under a CCO license.

Table 1 Statistical table of gene region and type of SNP

\begin{tabular}{lllllll}
\hline Sample-name & Downstream & Exonic & Intergenic & Intronic & Upstream & Total $^{a}$ \\
\hline Huangguazui(B1) & $1019480.70 \%$ & $992170.68 \%$ & $786538154.21 \%$ & $615179242.40 \%$ & $881760.61 \%$ & 14509223. \\
Wuhuatou(B2) & $977970.72 \%$ & $965300.71 \%$ & $740750054.23 \%$ & $578202342.33 \%$ & $848550.62 \%$ & 13660201. \\
\hline
\end{tabular}

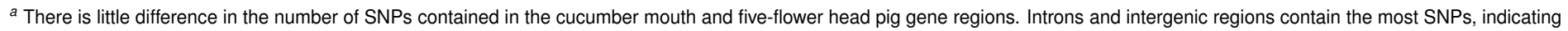
that the two regions have the most variation.

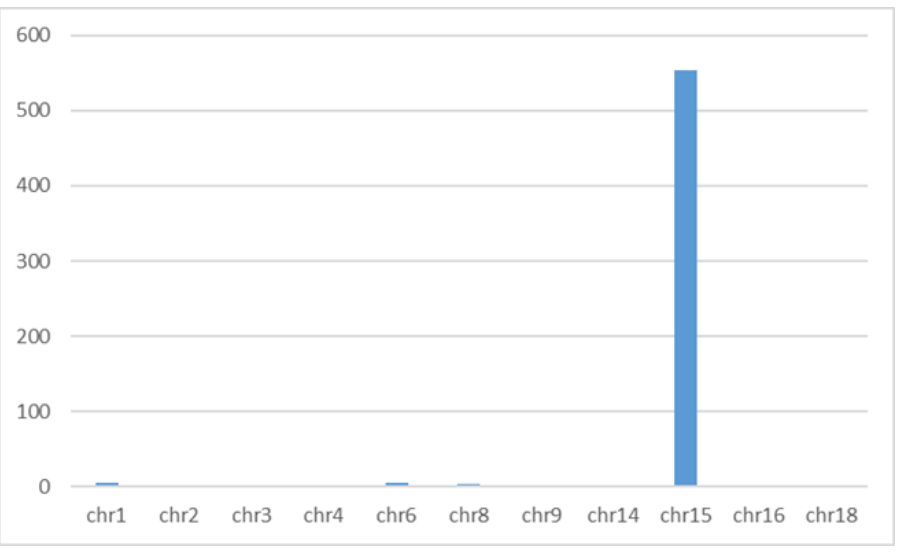

Figure 1 The abscissa represents the chromosome number, and the ordinate represents the number of SNPs. The distributions of the quantities and densities of the SNPs on the chromosomes were observed to not be uniform. For example, Chromosome 15 was short in length but had the largest number of SNPs, which was far more than the other examined chromosomes. These findings indicated that the densities of the SNPs on that particular chromosome were the highest. Therefore, it was considered that there was a relatively high density of variation on Chromosome 15. distance between the two strains in their genetic relationship.It was also determined in the phylogenetic tree analyses of the Shenxian pigs, large white pigs representative of introduced pigs, and Meishan pigs representative of local south China pigs, that the three populations had originated from the same species, and there was a distant genetic relationship between the Shenxian pigs and the other two examined populations.

The PCA analysis of the effective data obtained after quality controls were implemented were conducted using Plink software, and then the PCA analysis results were visualized using R software. In order to reveal the population specificity of the Huangguazui and Wuhuatou pigs, the SNP information of the two populations was analyzed using a principal component analysis (PCA) method. The top three eigenvectors were selected for the analysis, and the analysis results were visualized by $\mathrm{R}$ language, as shown in Fig. $2-3$. The analysis results of the first and second eigenvectors are detailed in Figure 3. It can be seen in the figure that the first eigenvector had accurately distinguished the Huangguazui and Wuhuatou into two strains, which confirmed that the differentiation among the Shenxian pig population was serious. The second eigenvector revealed that both the Huangguazui and Wuhuatou subpopulations were stratified within the total population. The analysis results of the first and third eigenvectors showed that the stratification in Huangguazui population was the most obvious (Figure 4). The second and third eigenvectors showed (Figure 5) that the degrees of stratification in the Wuhuatou strain were less

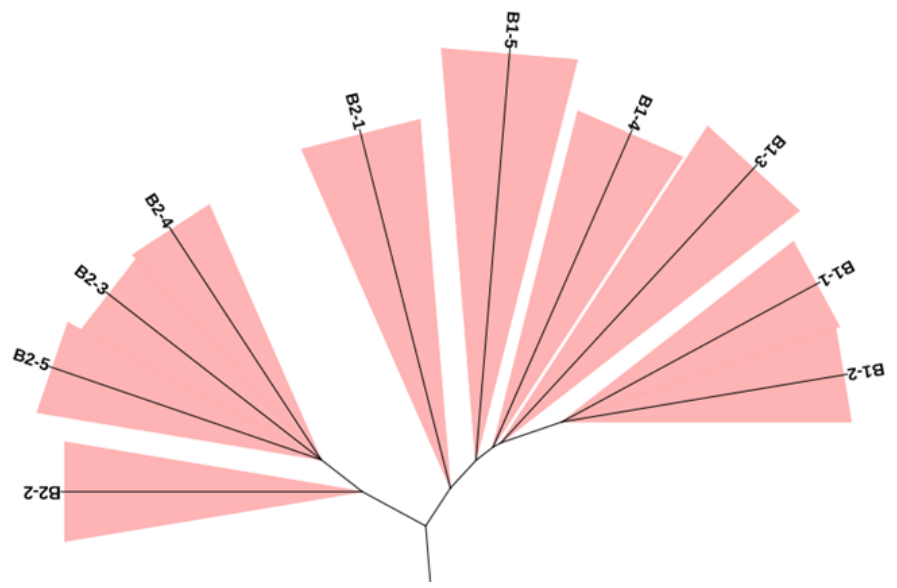

Figure 2 The analysis results showed that the Huangguazui and Wuhuatou subpopulations had originated from the same ancestor and belonged to the same variety. However, they could clearly be divided into two distinct groups, which indicated that there were indeed differences in the genetic backgrounds of the Huangguazui and Wuhuatou pigs. It was confirmed that there was a certain distance between the two strains in their genetic relationship.

than those of the Huangguazui strain.

\section{Discussion}

For DNA extraction and detection, the complete DNA in the agarose gel should be a band. At times, there will be three bands observed, which may have been formed by DNA degradation. Generally speaking, the foremost band is of supercoiled configuration; middle band is linear; and the last band is of single-chain defective configuration(Martina et al.2020). If mechanical damages occur during the extraction process, the DNA can become degraded, which may be presented as a diffuse state on the gel.

SNP mainly refers to the DNA sequence polymorphism caused by a single nucleotide variation at the genomic level. In this study, the SNP information obtained via statistical variation detection showed that the quantity and density distributions of the SNPs in the two subpopulations were uneven, and they were mainly concentrated on Chromosome 15. There have been many studies conducted regarding the QTL of meat quality traits on Chromosome 15(Liu.2015;Jia.2004), which may be related to the excellent meat quality traits of the Shenxian pig species(Hu et al.2015). Previous studies have successfully mapped the QTLs of the meat quality traits and acquired linked SNPs for labelling(Yan et al.2017; Li et al.2010;Science.2016b). However, the current SNP chip technology is not adequately developed for mapping the quantitative traits in pigs(Chen et al.2011). Therefore, the SNP and InDel information on Chromosome 15 will be detected, annotated, and analyzed in follow-up experimental research. 


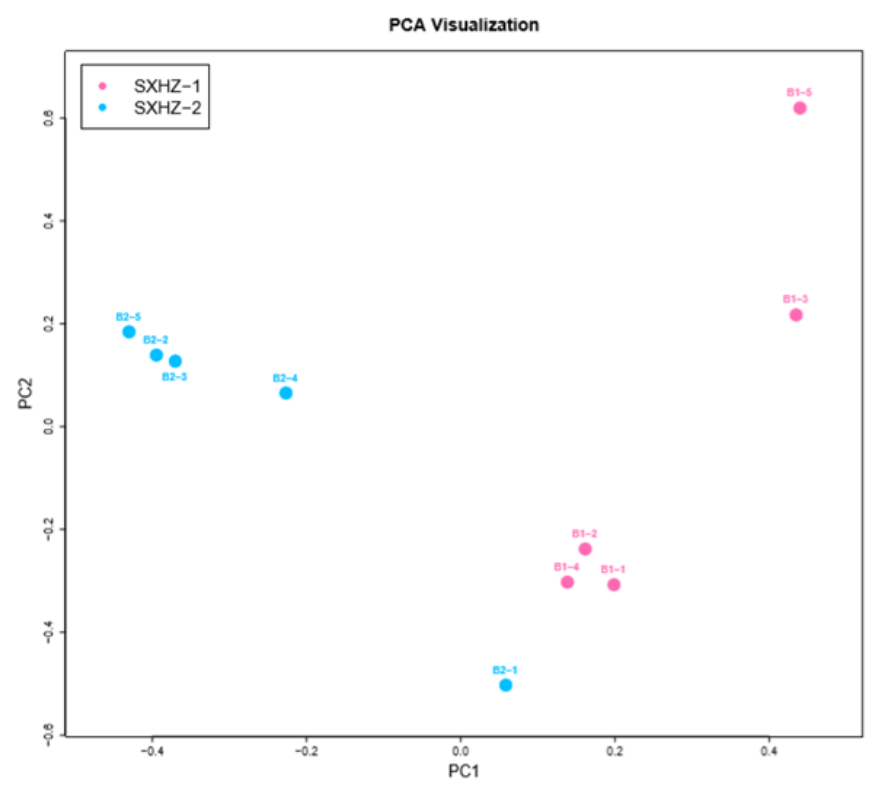

Figure 3 The abscissa represents the first vector, and the ordinate represents the second vector; pink represents cucumber-mouthed pigs, blue represents five-flowered pigs.

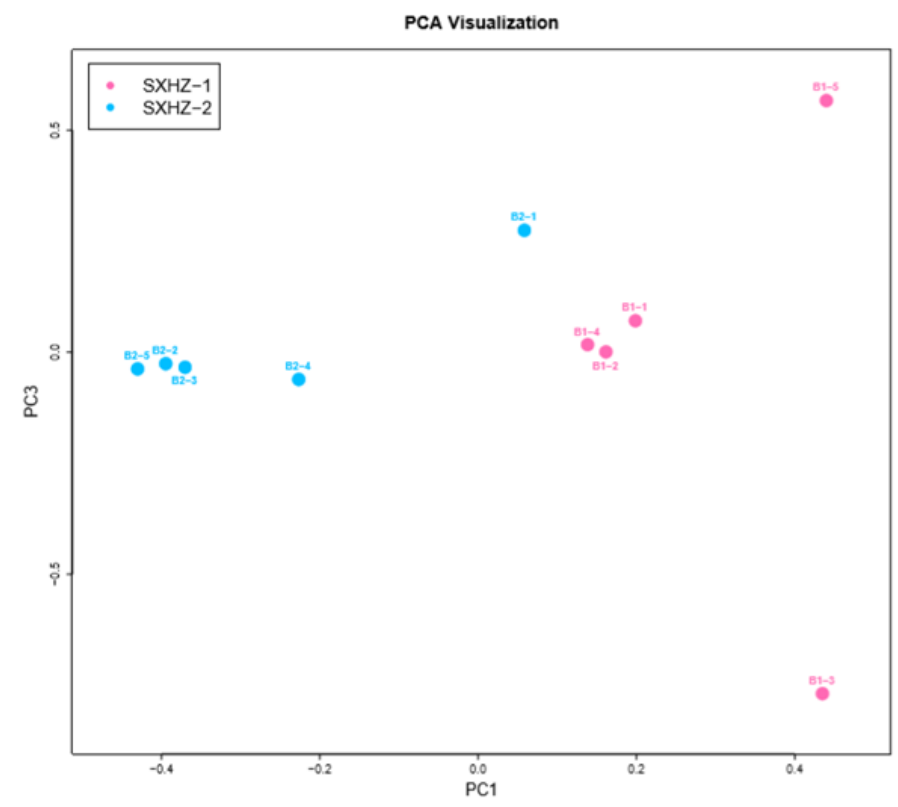

Figure 4 The abscissa represents the first vector, and the ordinate represents the third vector; pink represents cucumber-mouthed pigs, blue represents five-flowered pigs.

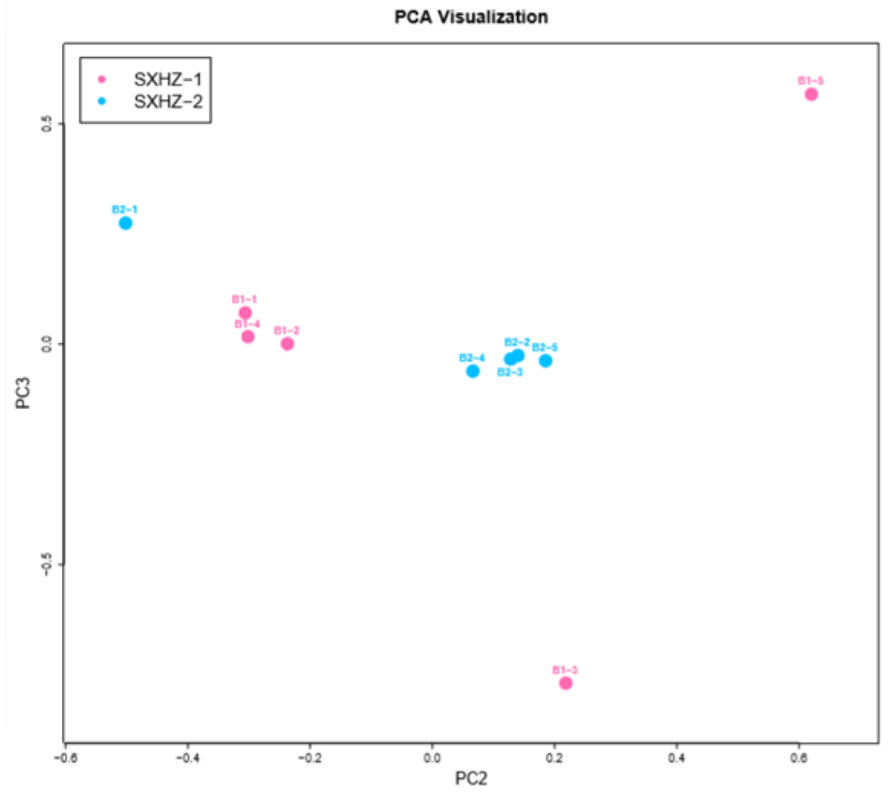

Figure 5 The abscissa represents the second vector, and the ordinate represents the third vector; pink represents cucumber-mouthed pigs, blue represents five-flowered pigs.

Furthermore, functional gene annotations will also be carried out in order to explore the differential genes, along with the quality traits regulated by the differential genes, in the two subpopulations of Shenxian pigs. Another area to be explored in the future will be the main traits controlled by Chromosome 15 in order to provide a research basis for the follow-up breeding of Shenxian pigs.

The whole genome population structure analysis processes are utilized to re-sequence different geographic locations and populations, as well as to obtain the SNP and other information after comparisons are made with the reference genome. Subsequently, the principal components and population structures among populations are analyzed, and appropriate phylogenetic trees are constructed. In the present study, based on the analysis results of the population structures of the examined species, the differences in the members of the examined species could be more clearly understood. It was found that the genome-wide population structure analysis results had inferred the characteristics, population structure, and development history of the varieties from the genome. The obvious stratification and severe phenotypic differentiation observed in the population structures of the Shenxian pigs indicated that the phenotypic traits of Huangguazui and Wuhuatou may have been less affected by artificial selection processes.

\section{LITERATURE CITED}

Changwei Bi, Na Lu, Zhen Huang, et al.,2020 Whole-genome resequencing reveals the pleistocene temporal dynamics of branchiostoma belcheri and branchiostoma floridae populations. Ecology and Evolution 10(15), 1-15.

Ley,T.J.,Mardis,E.R.,Ding,L.,et al.,2008 DNA sequencing of a cytogenetically normal acute myeloid leukaemia genome.Nature 456(7218):66-72.

Levy,S. E.,Myers, R. M.,2016 Advancements in next-generation sequencing. Annu Rev Genomics Hum Genet 17(1), 95-115.

Slatko Barton E, Gardner Andrew F, Ausubel Frederick M.,2018 
Overview of Next-Generation Sequencing Technologies122(1):e59.

Mardis,E.R.,2008 The impact of next-generation sequencing technology on genetics.Trends Genet 24:133-141.

Andersson,L.,2009 Genome-wide association analysis in domestic animals:a powerful approach for genetic dissection of trait loci.Genetica 136:341-349.

Mckenna,A., M.Hanna, E.Banks, et al.,2010 The Genome Analysis Toolkit: a MapReduce framework for analyzing next-generation DNA sequencing data.Genome Res 20:1297-1303.

Montgomery,S.B., D.L.Goode, E.Kvikstad, el al.,2013 The origin, evolution, and functional impact of short insertion-deletion variants identified in 179 human genomes. Genome Res 23:749-761.

Li.H.,J.Ruan,R.Durbin.,2008 Mapping short DNA sequence reads and calling variants using mapping quality scores.Genome Res 18:1851-1858.

Depristo,M.A.,Banks,E.,Poplin,R.et al.,2011 A framework for variation discovery and genotyping using next-generation dna sequencing data. Nature Genetics 43(5), 491-498.

Albers,C.A.,Lunter,G.,Macarthur,D. G.,2011 Dindel: accurate indel calls from short-read data. Genome Research 21(6), 10-11.

Li Heng, Handsaker Bob, Wysoker Alec, et al.,2009 The Sequence Alignment/Map format and SAMtools.. 25(16):2078-9.

Daniel C. Koboldt, Ken Chen, Todd Wylie, et al.,2009 VarScan: variant detection in massively parallel sequencing of individual and pooled samples 25(17):2283-2285.

Shan Gao, Jianhong Ou, Kai Xiao.,2014b R language and Bioconductor bioinformatics application. Tianjin: Tianjin Science and Technology Translation Publishing Company, 2014.

Kalyan Sudhaka.,2018b Python vs. R Programming Language. 8(8):70-79.

Kai W,Mingyao L,Hakon H.,2014 ANNOVAR:functional annotation of genetic variants from high-throughput sequencing data[J].Nucleic Acid Research.16(38):e164.

Group,T.I.S.M.W.,2001 A map of human genome sequence variation containing 1.42 million single nucleotide polymorphism. Nature 15.

Purcell S, Neale B, Todd-Brown K, et al.,2007 PLINK: a tool set for whole-genome association and population-based linkage analyses[J].Am J Hum Genet81(3): 559-575.

Martina Leonardi, Giulia La Marca, Barbara Pajola, et al.,2020 Assessment of real-time PCR for Helicobacter pylori DNA detection in stool with co-infection of intestinal parasites: a comparative study of DNA extraction methods. 20(1):193-213.

Liu Xianxian.,2015 QTL analysis on pig chromosome 15 affecting glycogen content and drip loss of longissimus dorsi muscle. Jiangxi Agricultural University.

Jia Chao.,2004 Study on the correlation between the quality traits of Sutai pork and microsatellite markers on chromosome 15.Nanjing Agricultural University.

Yingxin Hu,Suqiao Chu,Chunlian Lu,et al.,2015 Study on the characteristics of pig germplasm in Shenxian County.Animal Husbandry and Veterinary Medicine47(07):58-60.

Yan Guorong, Qiao Ruimin, Zhang Feng, et al.,2017 ImputationBased Whole-Genome Sequence Association Study Rediscovered the Missing QTL for Lumbar Number in Sutai Pigs.7(1):615.

Li HD, Lund MS, Chritensen OF, et al.,2010 Quantitaive trait loci analysis of swine meat quality traits.J Anim Sci 88(9): 2904-12.

Science,2016 Reports from China Agricultural University Advance Knowledge in Science (Identification of genes for controlling swine adipose deposition by integrating transcriptome, wholegenome resequencing, and quantitative trait loci data).
Chen Huiyong, Luo Saiqun, Wu Zhenfang.,2011 Current status of pig genome-wide SNP research. China High-tech Industrialization Research Association. Cultivating bio-industry and developing green economy_— The Fifth China Bio-industry Conference-2011 Gene Science and Industrial Development Forum Proceedings. China High-Tech Industrialization Research Association: China High-Tech Industrialization Research Association:4. 